\title{
A method to include plastic anisotropy to orthogonal micromachining of fec single crystals
}

\author{
Eralp Demir
}

Received: 14 October 2007 / Accepted: 28 August 2008 / Published online: 19 September 2008

(C) The Author(s) 2008. This article is published with open access at Springerlink.com

\begin{abstract}
The purpose of this study is to explain the experimentally observed differences in the machined surface quality and cutting forces of single crystals with the change in cutting direction and/or cutting plane with respect to the crystal orientation. Taylor-based perfectly plastic model is used to relate the crystallographic orientation to the cutting forces and the specific energy considering friction between the chip and the rake face of the tool. The model is valid for the depths of cuts that are greater than the limit below which size scale effects are observed $(\sim 1 \mu \mathrm{m})$. The periodic force and shear angle variations in the microtome experiments of Black et al. (Von Turkovich and Black, Trans Am Soc Mech Eng 92:130-134, 1970; Black, J Eng Ind 101:403-415, 1979; Cohen and Black, 1984) and the change in force and surface quality with a change in turning axis of a single crystal copper and aluminum in diamond turning experiments of To, Lee, and Chan (To et al., J Mater Process Technol 63:157-162, 1997) can be explained with the results of the model.
\end{abstract}

Keywords Taylor factor • Crystal plasticity • Orthogonal cutting $\cdot$ Micromachining •

Single crystal fcc $\cdot$ Cutting specific energy

E. Demir $(\varangle)$

Max-Planck-Institut fur Eisenforschung, Max-Planck-Str. 1, 40237 Düsseldorf, Germany

e-mail: e.demir@mpie.de

\section{Introduction}

It is important to know how good the quality of the product will be prior to machining. In other words, what would be the surface quality during planning if the workpiece is machined along a certain cutting direction compared to machining other directions? Experimental measures of cutting force, specific energy, chip morphology, and shear angle are used as references to give a wealth of information about the machined product.

Cutting tools of sizes as small as $25 \mu \mathrm{m}$ have become commercially available with the recent developments in tool manufacturing technology. Tools having sizes close to the sizes of grains give rise to a demand for understanding of crystallographic differences during machining processes [5].

Numerous studies on both single and polycrystal metals have elucidated the effect of the crystal orientation of the workpiece on the quality surface machined at small scales $[6,7,10-25]$. Understanding the difference in cutting forces for planning different orientations in the crystals has been a fundamental issue even though orthogonal planning is the simplest deformation case. In addition, few modeling efforts exist in the literature that relate the crystal orientation to the machining forces and surface quality [26-28]. There has not yet been an explanation for the very low shear angles observed that is against Merchant minimum energy principle when machining single crystals $[3,8,9]$.

Planning and turning (assuming zero side and back rake angles for turning) are orthogonal cutting operations. Orthogonal machining is the simplest machining operation, and it could be easily extended to the other conventional machining processes like milling and drilling. It is a plane strain deformation where the 
width of cut is significantly large compared to the depth of cut. The deformation is of simple shear type, and the amount of shear strain, $\gamma$, increases with decreasing rake angle, $\alpha$ in Eq. 1.

$\gamma=45-\frac{\alpha}{2}$

In planning operation, the crystal orientation remain the same throughout the machining process, Fig. 1. In Taylor-based models, the Taylor factor, which is a measure of work done on the crystal, remains constant in the case of planning since the crystal orientation does not change. However, in turning operation, the crystal rotates around a prescribed crystallographic plane, Fig. 2. The cutting plane remains constant and cutting direction changes its direction during one revolution of the workpiece.

According to shear plane assumption, primary shear occurs on a single plane [29]. However, measurements have shown that this assumption is rather strict, especially for small-scale cutting operations. For instance, three different deformation zones are observed when machining aluminum rather than deformation being restricted to a single plane [8].

Friction lowers the shear angle, $\phi$, (Merchant's relation), Eq. 2. The friction angle, $\beta$, between the tool rake face and the workpiece material is included in the plasticity model by reducing the possible range of shear angles. This in turn eliminates very high shear angles that the crystal plasticity model could yield. However, the dependence of friction coefficient on the crystallographic orientation is known [9]. The change in the type

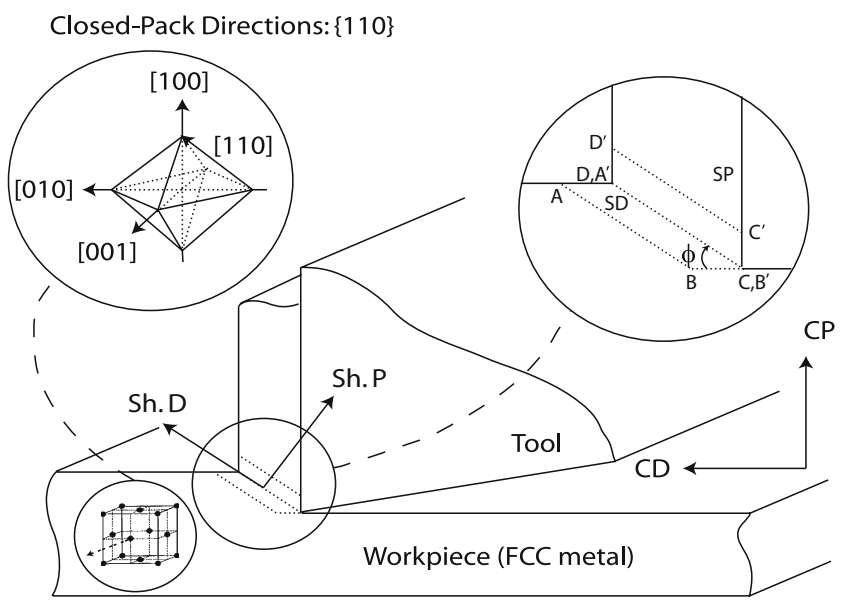

Fig. 1 Planning fcc crystal. Sh.P, shear plane; Sh.D, shear direction; $C P$, cutting plane; $C D$, cutting direction

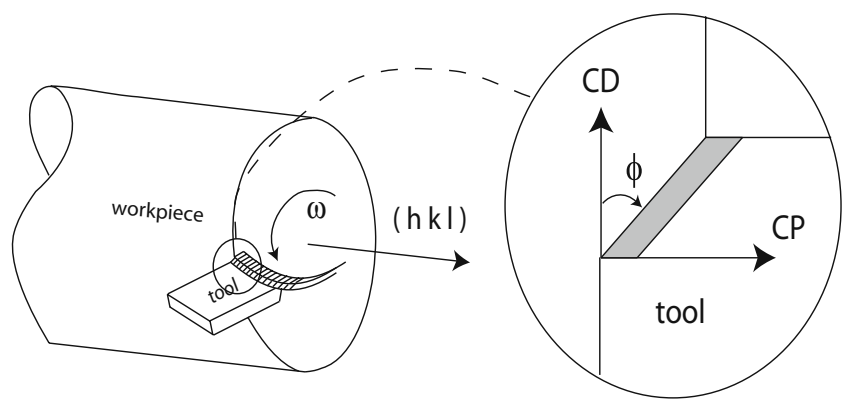

Fig. 2 Turning fcc crystal around a crystallographic plane

of the chip varies the contact area between the chip and rake face of the tool, as well as the friction coefficient.

$\phi=45+\frac{\alpha}{2}-\frac{\beta}{2}$

Edge radius and initial material dislocation content, depending on its strain history, limit the observation of crystallographic effects during machining $[30,31]$. Lucca developed a sliding indentation model and estimated subsurface damage for aluminum to be $0.31-0.81 \mu \mathrm{m}$ for cut depths of $0.1-1 \mu \mathrm{m}$, respectively, that match with the X-ray measurements [32]. Moriwaki and Okuda [25] observed that, below a $0.2-\mu \mathrm{m}$ depth of cut, rubbing and burnishing become more dominant than shear and no crystallographic effect is found while machining polycrystal copper.

The yield behavior during machining slightly depends on the rate of deformation. As the strain rate increases, the cutting resistance also increases and the shear zone gets smaller and deformation becomes constricted to a narrower region for shorter time intervals [33].

Taylor-based models can be used to explain crystallographic differences if (1) the ploughing effect between the tool and the workpiece, (2) the edge radius, and (3) the strain rate effects are ignored. In contrast to the uniqueness problem of rate-insensitive models $[34,37]$, a unique solution can be found for the amount of work and the specific energy. The specific energies or forces can be obtained for deformations with complicated tool geometries with the use of Taylor's models easily. Taylor-based models, having no need for iterative nonlinear solvers, are simpler than ratesensitive models.

In this paper, a Taylor-based crystal plasticity model is used to explain experimentally observed crystallographic effects on orthogonal machining of single-crystal materials. Initially, a brief description of Schmid- and Taylor-based models is presented, and finally, the Taylor method is used to estimate shear angles and forces for orthogonal machining processes. 
The method is applied to various crystal orientations for turning and planning.

\section{Model and results}

The application of Schmid- and Taylor-based models to orthogonal machining processes is discussed in the following sections. The Taylor-based model is implemented in MatLab.

\subsection{Schmid-based model}

In previous studies, Schmid-based models are used to find the shear angle by using measured thrust and cutting forces and by resolving those forces into slip systems [8]. There are two major drawbacks of these models. First, Sato and coworkers assumed that the amount of slip is proportional to the amount of resolved shear stress, $\tau_{R S S}$, which is not in agreement with the Schmid theory ${ }^{1}$ [37]. Second, material simply having a principal stress state is even rougher assumption than the shear plane assumption, which would have a stress state at the shear plane, $\sigma^{\text {shear }}$, having a shear, $\tau_{\mathrm{s}}$, and a normal stress component, $\sigma_{\mathrm{n}}$, Eq. 3 .

$\sigma^{\text {shear }}=\left(\begin{array}{ccc}0 & 0 & \tau_{\mathrm{s}} \\ 0 & 0 & 0 \\ \tau_{\mathrm{s}} & 0 & \sigma_{\mathrm{n}}\end{array}\right)$

Schmid-based models need three main inputs; the stress state, the crystal orientation with respect to the applied stress, and the critical threshold stress, $\tau_{\mathrm{cr}}$, at which yielding starts. Therefore, the experimentally determined forces and the shear angle have to be used as inputs to the model in order to determine the stress state at the shear plane. The estimated shear angle can be found by using seven steps: (1) assume a shear angle between $1^{\circ}$ and $88^{\circ}$; (2) transform the stress state from the shear plane to the sample frame and then to the crystal frame; (3) resolve the stresses into the 12 slip systems to find $\tau_{\text {RSS }}$ using Eq. 4 , where $b$ and $n$ are the slip direction and slip plane, respectively; ${ }^{2}(4)$ assume a value for critical threshold stress $\left(\tau_{\mathrm{cr}}\right)$ and identify the active slip systems if $\tau_{\text {RSS }}$ exceed that value; (5) sum all the active slip vectors to get the resultant slip vector; (6) transform the resultant vector from the crystal frame

\footnotetext{
${ }^{1}$ The active slip systems are the ones that exceed the critical value of shear stress when the applied stress is resolved to the 12 slip systems according to Schmid.

${ }^{2}$ Subscripts indicate the tensor components in index notation.
}

to the sample frame; and (7) identify the components of the resultant slip vector along the cutting direction and the cutting plane and estimate the shear angle. ${ }^{3}$

$\tau_{\mathrm{RSS}}=b_{i} \sigma_{i j} n_{j}$

\subsection{Taylor-based model}

This model is based on Taylor's multiple slip analysis for single crystals. Five slip systems are assumed to be active for the given deformation among 12 since strain has five independent unknowns assuming constant dilatation during deformation.

According to Taylor, the shears along each of the five slip systems are the ones that minimize the sum of internal work done in the crystal. There are two inputs to the model: (1) the deformation gradient and (2) the crystal orientation with respect to the given deformation gradient. The five slip systems selected must be geometrically independent.

The rate-insensitive methods posses an ambiguity problem in selecting the active slip systems. However, a unique solution can be found for the amount of work, which is of interest to us.

The shear angle and the shear stress at the shear plane are calculated by minimizing the crystallographic work. This is achieved by searching through a range of possible shear angles for a given crystal orientation. In order to illustrate changes that are purely crystallographic, no kinematic constraints are introduced to the shear angle even though values greater than $60^{\circ}$ are not likely to be found. The range used for the shear angles is between $1^{\circ}$ and $88^{\circ}$ in this study.

In plane strain orthogonal cutting, the deformation gradient $\left(\mathrm{DG}^{\mathrm{sh}}\right)$ on the shear plane, consists of two parts; the shear strain part $\left(\epsilon^{\text {sh }}\right)$ and the rotation $\operatorname{part}\left(\Omega^{\text {sh }}\right){ }^{4}$

$\mathrm{DG}^{\mathrm{sh}}=\epsilon^{\mathrm{sh}}+\Omega^{\mathrm{sh}}$

$\mathrm{DG}^{\mathrm{sh}}=\left(\begin{array}{lll}0 & 0 & \gamma \\ 0 & 0 & 0 \\ 0 & 0 & 0\end{array}\right)=\left(\begin{array}{lll}0 & 0 & \frac{\gamma}{2} \\ 0 & 0 & 0 \\ \frac{\gamma}{2} & 0 & 0\end{array}\right)+\left(\begin{array}{ccc}0 & 0 & \frac{\gamma}{2} \\ 0 & 0 & 0 \\ -\frac{\gamma}{2} & 0 & 0\end{array}\right)$

Taylor's model uses the symmetric part of the DG that is the strain part as an input only. The shear strain must be transformed from the shear plane to the sample

\footnotetext{
${ }^{3}$ Simply projecting the resultant vector to the cutting direction would yield a spatial angle rather than the shear angle defined in accordance with the shear plane assumption since the resultant vector is a spatial vector.

${ }^{4}$ The superscript "s" refers to the sample frame that is defined with the cutting direction and plane. The superscripts "sh" and "c" indicate the shear plane and the crystal frame, respectively.
} 
frame, to find the strain at the sample frame, $\epsilon^{\mathrm{s}}$, through the assumed shear angle of $\phi$ with the transformation $g^{\text {sh2s }}$, Eq. 7.

$g^{\mathrm{sh} 2 \mathrm{~s}}=\left(\begin{array}{ccc}\cos \phi & 0 & -\sin \phi \\ 0 & 1 & 0 \\ \sin \phi & 0 & \cos \phi\end{array}\right)$

$\epsilon_{i j}^{\mathrm{s}}=g_{i k}^{\mathrm{sh} 2 \mathrm{~s}} g_{j l}^{\mathrm{sh} 2 \mathrm{~s}} \epsilon_{k l}^{\mathrm{sh}}$

Equation 9 shows the transformation matrix for the given cutting direction of [uvw] and cutting plane of (hkl). Shear strain has to be transformed from the sample to the crystal frame with the transformation rule shown in Eq. 10 to perform work calculation.

$g^{\mathrm{s} 2 \mathrm{c}}=\left(\begin{array}{ccc}u & o & h \\ v & p & k \\ w & q & l\end{array}\right)$

$\epsilon_{i j}^{\mathrm{c}}=g_{i k}^{\mathrm{s} 2 \mathrm{c}} g_{j l}^{\mathrm{s} 2 \mathrm{c}} \epsilon_{k l}^{\mathrm{s}}$

There are two ways to estimate the crystallographic work. One way is to multiply microscopic shears, $\Gamma$ (Table 1), with the slip resistances of $\tau_{\mathrm{cr}}$ at each slip system and sum for five slip systems, $\alpha$, and minimize the work, dW [34]. Another way is to find the nearest stress state among the 28 macroscopic stress states to the given strain in the crystal that maximizes the work [35].

$\mathrm{dW}=\sigma \epsilon^{\mathrm{c}}=\tau_{\mathrm{cr}}^{\alpha} \Gamma^{\alpha}$

Five unknown shear increments, $d \gamma^{\alpha}$, are calculated through a five-by-five coefficient matrix, $m^{\alpha}$, formed for every five of the 12 systems, making 792 slip system combinations. The five systems are checked against geometrical dependence and the shear increments at the glide plane $\alpha, d \gamma^{\alpha}$, are estimated from the given transformed state of strain in the crystal, $\epsilon^{\mathrm{c}}$.

$\epsilon_{i j}^{\mathrm{c}}=\sum_{\alpha=1}^{5} m_{i j}^{\alpha} d \gamma^{\alpha}$

$m_{i j}^{\alpha}=\sum_{\alpha=1}^{5} b_{i}^{\alpha} \otimes n_{j}^{\alpha}$
The solution is determined by minimizing work through Eq. 14. Minimizing work is equivalent to minimizing the sum of shears assuming equal hardening for all of the slip systems.

$\mathrm{dW}=\tau_{\mathrm{cr}} \sum_{\alpha=1}^{5} d \gamma^{\alpha}$

Taylor factor, $M$, is a measure of work done on the crystal for a given orientation and deformation. It is the ratio of the applied stress to critical resolved shear stress, $\tau_{\mathrm{cr}}$.

$M=\frac{\sigma}{\tau_{\mathrm{cr}}}=\frac{\sum_{\alpha=1}^{5} d \gamma^{\alpha}}{\epsilon_{v m}}$

In the case of machining, the work done by the slip deformation is equivalent to the work done on the shear plane $\left(\epsilon_{v m}=\frac{\gamma}{2}\right.$ and $\left.\sigma=\tau_{\text {sh }}\right)$. Taylor factor then reads

$M=\frac{\sum_{\alpha=1}^{5} d \gamma^{\alpha}}{\frac{\gamma}{2}}=\frac{\mathrm{dW}}{\tau_{\mathrm{cr}} \frac{\gamma}{2}}$

The macroscopic work, $\mathrm{dW}$, in terms of total shear, $\gamma$, becomes, according shear plane assumption,

$\mathrm{dW}=\tau_{\mathrm{sh}} \frac{\gamma}{2}$

The shear stress on the shear plane, $\tau_{\mathrm{sh}}$, can be related to Taylor factor using the equality between the macroscopic and the microscopic works.

$\tau_{\mathrm{sh}}=M \tau_{\mathrm{cr}}$

Figure 3 shows the results for turning case for three different crystallographic axes without friction. The Taylor factor variation is due to the change of crystal orientation during one revolution of the workpiece. The fluctuation in the Taylor factor (or in the amount of crystallographic work) is significant for cutting planes of (110) and (111). The Taylor factor shows a smooth variation when (100) is the cutting plane, meaning less force fluctuations and better surface quality are expected compared to the cutting planes of (110) and (111), similar to the experimental observations of Lee and To [4].

Figure 4 shows the Taylor factors estimated in the case of planning for a given cutting direction and plane.

Table 1 Twelve slip systems for FCC metals

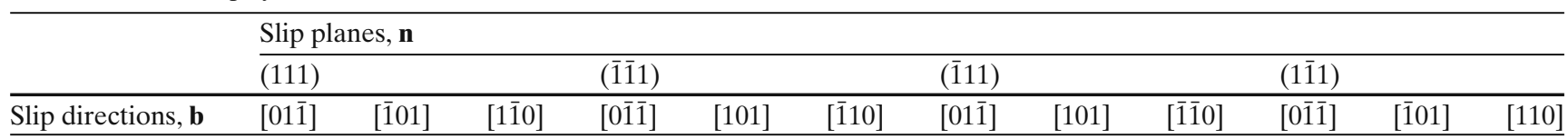




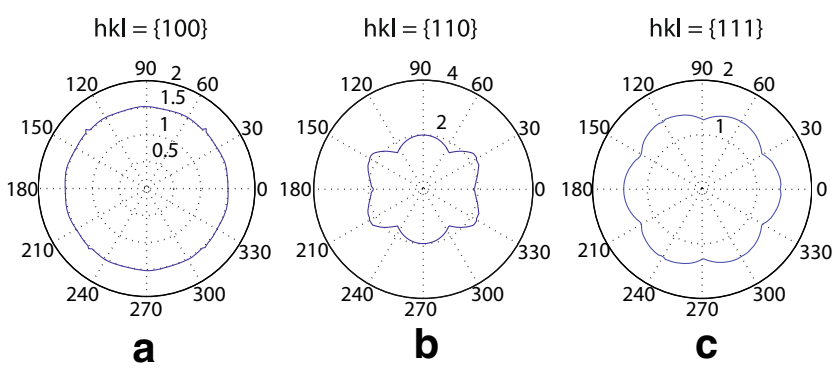

Fig. 3 Taylor factor variations for turning around the cutting planes (CP) of a (100), b (110), and $\mathbf{c}(111)$

The orientation transformation matrix, $g^{\mathrm{s} 2 \mathrm{c}}$, is obtained through Eq. 9 with Euler angles in Bunge convention. Every point in each of the plots belongs to a colatitude $\left(\phi_{1}\right)$ and longitude $(\Phi)$ for a constant $\phi_{2}$. Over $50 \%$ change in work emphasizes the existence of strong crystallographic anisotropy between different orientations.

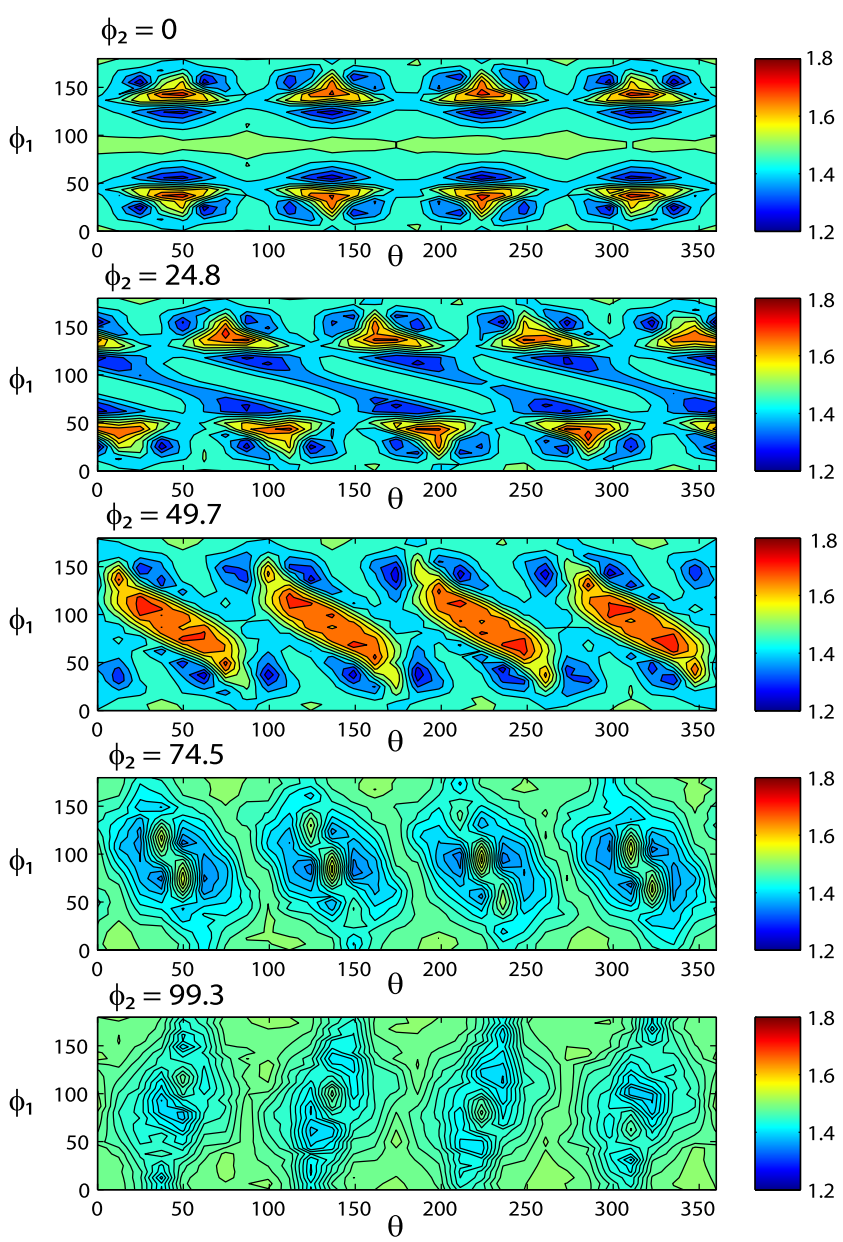

Fig. 4 Taylor factor for orthogonal machining for orientations defined with Euler angles $\left(\phi_{1}, \theta, \phi_{2}\right)$ without friction
Besides, for certain orientations, the change in Taylor factor is much less (last two rows in Fig. 4).

The cutting specific energy, $K_{\text {cut }}^{\mathrm{sp}}$, defined as the cutting force divided by the uncut chip thickness ( $d$ : depth of cut, $w$ : width of cut, $A^{\text {sh }}$ : sheared area), is estimated in terms of the Taylor factor. The normal stress at the shear plane is assumed be one third of the hardness, $H$ [38]. The cutting force, $\mathrm{Fc}$, is then calculated knowing the shear angle, the shear stress, and the normal stress at the shear plane.

$\mathrm{Fc}=K_{\mathrm{cut}}^{\mathrm{sp}}(d w)=\left(\tau_{\mathrm{sh}} \cos \phi-\frac{H}{3} \sin \phi\right) A^{\mathrm{sh}}$

where the sheared area

$A^{\mathrm{sh}}=\frac{d w}{\sin \phi}$

The specific energy then becomes

$K_{\mathrm{cut}}^{\mathrm{sp}}=\frac{\tau_{\mathrm{sh}}}{\tan \phi}-\frac{H}{3}$

Substituting the shear stress from Eq. 18, specific energy can be obtained in terms of the Taylor factor, the shear angle, the critical resolved shear stress, and the hardness, Eq. 22.

$K_{\mathrm{cut}}^{\mathrm{sp}}=\frac{M \tau_{\mathrm{cr}}}{\tan \phi}-\frac{H}{3}$

Figure 5 shows the change of specific energy when planning different orientations as the workpiece is rotated around [100] and planned successively. Note that

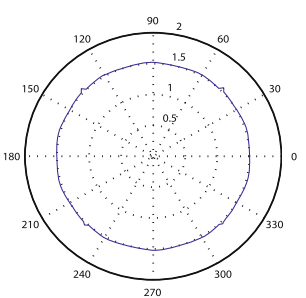

a.

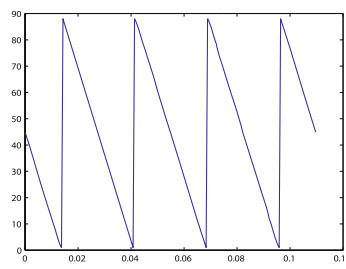

C.

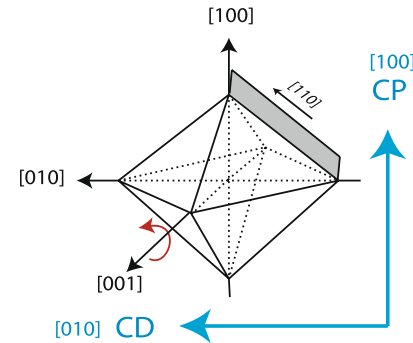

b.

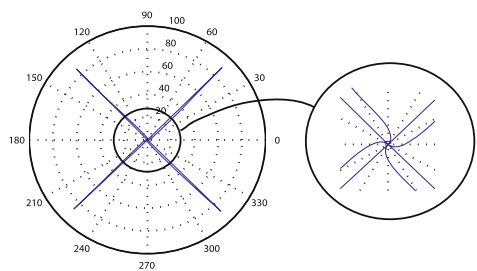

d.
Fig. 5 a Taylor factor change; $\mathbf{b}$ crystal orientation and the closed pack directions in the crystal; c shear angle change; d specific energy without friction when the workpiece rotates $10^{\circ}$ around [100] direction after each cut 


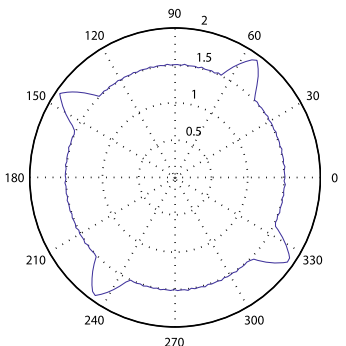

a.

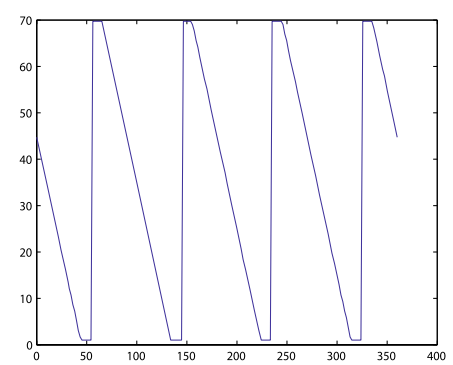

b.
Fig. 6 a Taylor factor; $\mathbf{b}$ shear angle results with assumed friction coefficient of 0.33 when the workpiece rotates around [100] direction after each cut

the crystal orientation is intact during planning. The Taylor factor for [100] remains constant because the input deformation gradient, which is of simple shear type, activates two slip systems that share the same slip direction, Fig. 5b, and work-minimization always yields the same couple of slip systems when the sample is rotated around [100] and a new cut is made. The specific energy takes quite large values when the shear angle is close to $0^{\circ}$, Eq. 22.

Friction angle is introduced into the model to avoid unreasonably high shear angles. Friction angle limits the possible range of shear angles through Eq. 2 and reduces the number of available slip systems. Figure 6 shows the model results for an assumed coefficient of friction of 0.33 between the tool rake-face and the
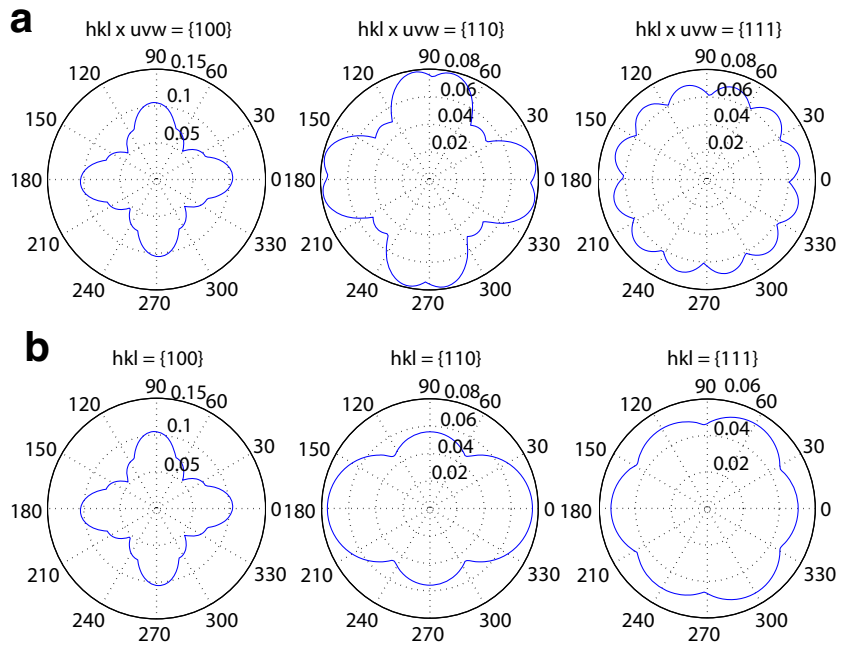

Fig. 7 Specific energy variations for a planning and $\mathbf{b}$ turning when the specific energy is minimized instead of crystallographic work

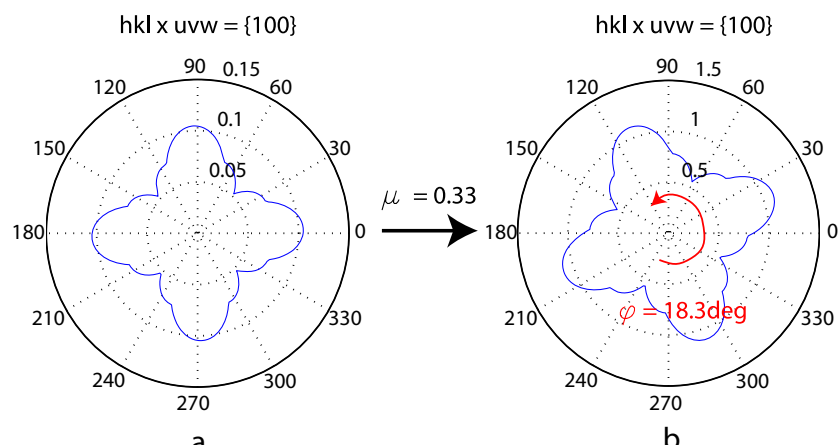

a.

Fig. 8 The effect of friction when specific energy is minimized a without friction and $\mathbf{b}$ with a friction coefficient of 0.33 for planning different orientations around $\{100\}$

workpiece that limits the range of shear angles to $69.7^{\circ}$. Friction acts as an upper physical constraint imposed to the shear angle. A fourfold symmetrical variation of Taylor factor and specific energy is observed very similar to microtome experiments of Black and Cohen for the same orientation [1-3].

\subsection{Minimization of cutting specific energy}

The specific energies can take unreasonably high values when the shear angles are very low (Eq. 22). Therefore, a more realistic solution for the specific energies is necessary. To achieve this, specific energy is minimized instead of minimizing plastic work described in the previous section, Eq. 23.

$\min \left\{\frac{M \tau_{\mathrm{cr}}}{\tan \phi}-\frac{H}{3}\right\}$

Figure 7 shows the results for different orientations for planning and turning with cutting direction and plane of $u v w$ and $h k l$, respectively. ${ }^{5}$ There is significant variation in the specific energy due to the plastic anisotropy of the fcc crystals.

Figure 8 shows the influence of friction on the resulting specific energy plots, a rotation of specific energy plots (the friction is introduced as outlined in Section 2.2).

The resulting plots for machining around the (100) plane can be used to explain the fourfold symmetrical changes in the measured shear angles and cutting forces in the experiments of Black and Cohen [1,2]. The

\footnotetext{
${ }^{5}$ The cutting plane $(\mathrm{CP})$ remains constant during turning.
} 
model has to be calibrated for the experimentally determined values of specific energies for different materials. anisotropy of the crystal for a given deformation geometry.

\section{Conclusions}

Several important conclusions are outlined as follows:

- The Taylor-based model clearly shows the effect of the crystal orientation of on the work and specific energy required to shear a crystal.

- Slip is a simple shear type of deformation on closed pack planes along closed pack directions [34-37]. The deformation gradient is of simple shear type for both the machining shear and the dislocation slip. Hence, work minimization always boils down to the problem of finding the nearest closed pack directions in a range of possible shear angles.

- Multiple numbers of slip systems are active during turning leading to higher magnitudes of Taylor factor and higher specific energies compared to planning.

- The model can be used to explain why Lee and coworkers [4] observed better surface quality when turning a crystal around (100) compared to the cutting planes of (110) and (111), Fig. 3.

- The fourfold symmetric fluctuations in the shear angle and forces observed in the orthogonal cutting experiments of Black and Cohen [1-3] can be explained with the friction or placing a constraint (upper limit) on the shear angle, Fig. 6. The minor force peaks experimentally observed at the tip of the major peaks are believed to be due to an interchange region in the active slip systems.

- Minimizing the crystallographic work yields unreasonably high specific energy and minimization of cutting specific energy gives reasonable specific energies while resulting in high shear angles. The real solution is believed to be in between the results of the two minimization schemes.

- Friction between the tool rake face and the chip depends on the type of chip formed [9] since the contact area changes. Therefore, the dependence of friction on the crystal orientation has to be included in the model.

- A kinematic constraint is necessary to avoid very large shear angles, which would have the same effect as the friction between the tool and the chip.

- The model can be easily extended further to other machining processes like milling to explain the part of the force fluctuations that is due to plastic

\section{Comments}

Some important comments for future studies are as follows:

- Application of symmetry to the measured force or shear angle data and extrapolating information for the other orientations in the case of planning is not suggested since the friction changes the axis of symmetry, Fig. 8.

- The minimization of work is equivalent to finding the nearest closed-pack directions in a crystal. Therefore, work minimization with the use of ratesensitive models together with phenomenological slip rate and hardening laws would give the same results as the rate-insensitive models.

- Crystal plasticity-based finite element models for machining processes with full description of boundary conditions would allow the most realistic modeling of machining processes.

- The finite element methods can explain the edge radius effects as the depth of cut gets smaller. However, no size scale effects, meaning no strengthening or increase in cutting specific energy, would be observed as the depth of cut gets smaller if the edge radius was kept the same.

- In order include size scale effects during machining, strain gradients have to be included into the finite element formulations. The rotational gradients of strains can be expressed in terms of geometrically necessary dislocation density, which contributes to the flow strength of the material. Therefore, nonlocal dislocation (state-variable) based models are essential in capturing size effects together with finite element crystal plasticity models.

Open Access This article is distributed under the terms of the Creative Commons Attribution Noncommercial License which permits any noncommercial use, distribution, and reproduction in any medium, provided the original author(s) and source are credited.

\section{References}

1. Von Turkovich BF, Black JT (1970) Micro-machining of copper and aluminum single crystals. Trans Am Soc Mech Eng 92:130-134 
2. Black JT (1979) Flow stress model in metal cutting. J Eng Ind 101:403-415

3. Cohen P, Black JT (1984) Strain, strain rate and shear velocity measurements in metal cutting. In: High energy rate fabrication. ASME, New York, pp 271-278

4. To S, Lee WB, Chan CY (1997) Ultraprecision diamond turning of aluminum single crystals. J Mater Process Technol 63:157-162

5. Van Luttervelt CA, Childs THC, Jawahir IS, Klocke F, Venuvinod PK (1998) Present situation and future trends in modeling of machining operations. CIRP 47:587-636

6. Koenig W, Spenrath N (1991) Influence of crystallographic structure of the substrate material on surface quality and cutting forces in micromachining. In: Proceedings of the international precision engineering seminar. pp 141-151

7. Williams JA, Horne JG, (1982) Crystallographic effects in metal cutting. J Mater Sci 17:2618-2624

8. Sato M, Kato Y, Tsutiya K, Aoki S (1980) Effects of crystal orientation on the cutting mechanism of aluminum single crystal. Bull JSME 24(215):1864-1870

9. Ueda K, Iwata K, Nakajama K (1980) Chip formation mechanism in single crystal cutting $\beta$-Brass. CIRP Ann - Manuf Technol 29(1):41-46

10. Yan J, Syoji K, Tamaki J (2004) Crystallographic effects in micro/nanomachining of single-crystal calcium floride. $\mathrm{J}$ Vac Sci Technol B 22(1):46-51

11. Lee WB (1990) Prediction of microcutting force variation in ultra-precision machining. Precis Eng 12(1):25-28

12. Zhou M, Ngoi BK (2001) Effect of tool and workpiece anisotropy on microcutting processes. IMechE J Eng Manuf 215:13-19

13. Lee WB, Cheung CF, To S (2002) A microplasticity analysis of micro-cutting force variation in ultra-precision diamond turning. Trans Am Soc Mech Eng 124:170-177

14. Lee WB, Chan KC (1990) Symmetry requirement in shear band formation. Scr Mater 24:997-1002

15. Lee WB, Zhou M (1993) A theoretical analysis of the effect of crystallographic orientation on chip formation in micromachining. Int J Mach Tools Manuf 33(3):439-447

16. Lee WB, To S, Sze YK, Cheung CF (2003) Effect of material anisotropy on shear angle prediction in metal cutting-a mesoplasticity approach. Int J Mech Sci 45:1739-1749

17. Lee WB, To S, Cheung CF (2000) Effect of crystallographic orientation in diamond turning of copper single crystals. Scr Mater 42:937-945

18. Lee WB, Cheung CF (2001) A dynamic surface topography model for the prediction of nano-surface generation in ultraprecision machining. Mech Sci 43:961-991

19. Cheung CF (2003) Influence of cutting friction on anisotropy of surface properties in ultra-precision machining of brittle single crystals. Scr Mater 48:1213-1218
20. Lee WB, To S, Cheung CF (2003) Friction-induced fluctuation of cutting forces in the diamond turning of aluminum single crystals. IMechE J Eng Manuf 217:615-631

21. Yuan ZJ, Lee WB, Yao YX, Zhou M (1994) Effect of crystallographic orientation on cutting forces and surface quality in diamond cutting of single crystal. CIRP Ann 43:39-42

22. To S, Lee WB, Chan CY (1997) Ultraprecision diamond turning of aluminium single crystals. Mater Process Tech 63:157-162

23. Blackley WS, Scattergood RO (1990) Crystal orientation dependence machining damage - a stress model. J Am Ceram Soc 73:3113-15

24. Ohmori G, Takada S (1982) Primary factors effecting accuracy in ultra-precision machining by dimamond tools. Bull Jpn Soc Precis Eng 16(1):3-7

25. Moriwaki T, Okuda K (1989) Machinability of copper in ultra-precision micro diamond cutting. CIRP Ann 38(1): $115-118$

26. Iwata K, Osakada K, Terasaka Y (1984) Process modeling of orthogonal cutting by the rigid-plastic finite element method. Trans Am Soc Mech Eng 106:132-138

27. Wince JN (2002) modeling chip formation in orthogonal metal cutting using finite element methods. Masters thesis to Mississippi State University, Mech. Eng. Dept.

28. Komanduri R, Chandrasekaran N, Raff LM (1999) Orientation effects in nanometric cutting of single crystal materials an MD simulation approach. CIRP Ann 48:67-72

29. Shaw MC, Finnie I (1955) Shear stress in metal cutting. Trans Am Soc Mech Eng 77:115-125

30. Liang Y, Moronuki N, Furukawa Y (1994) Calculations of the effect of material anisotropy on microcutting process. Precis Eng 16:132-138

31. Rubenstein C, Lau WS, Venuvinod PK (1985) Flow of workpiece materials in the vicinity of the cutting edge. Int J Mach Tool Des Res 25:91-97

32. Lucca DA, Seo A (1994) sliding indentatiion model of the tool-workpiece interface in ultra-precision machining. In: Tribology Symposium ASME. pp 17-22

33. Manjunathaiah J, Endres WJ (2000) A new model and analysis of orthogonal machining with an edge-radiused tool. Trans Am Soc Mech Eng 122:384-390

34. Taylor GI (1938) Plastic strain in metals. Inst Metals 62: 307-325

35. Bishop JFW, Hill R (1951) A theory of plastic distorsion of a polycrystalline aggregate under combined stresses. Phil Mag 42:1298-1307

36. Reid CN (1973) Deformation geometry of materials, 1st edn. Pergamon, Oxford

37. Kocks UF, Tome CN, Wenk HR (1998) Texture and anisotropy. Cambridge University Press, Cambridge

38. Arcona C, Dow TA (1998) An emprical tool force model for precision machining. Trans Am Soc Mech Eng 120:700-707 